\title{
Numerical simulation of micro-crack occurring in pipe made of stainless steel
}

\author{
Daria Wotzka ${ }^{1, *}$ \\ ${ }^{1}$ Opole University of Technology, 45-758 Opole, Poland
}

\begin{abstract}
Research works carried out regard to studies aiming at determination of the effect of cumulative duty operation on the development of micro-cracks in pipelines for transport of chemical substances. This paper presents results of computer simulations of a pipeline made of stainless steel. The model was investigated using the COMSOL Multiphysics environment. The object under study was divided into sub areas and then discretized according to the FEM method. The physico-chemical parameters of individual areas were defined based on measurement data. The main aim of research works was the modeling of acoustic emission wave, which is emitted in the vicinity of the tip of micro-crack as a result of its development. In order to solve the task, heterogeneity in the structure of the material, referred to damage/micro-crack, causing local stresses was assumed. The local stresses give rise to elastic waves, which propagate in the material in all directions. When the emission waves reach the boundaries of the pipe they are then transferred into acoustic waves and propagate in the surround air, until their natural attenuation. The numerical model takes into account the effect of high pressure (3.6 MPa) and negative temperature $\left(-100^{\circ} \mathrm{C}\right)$ of the gas, transported inside the pipe. The influence of changes of these values in the range of $\pm 20 \%$ on the obtained results was investigated. The main contribution of the works is the multiphysical simulation model of transportation pipe made of steel, coupling structural mechanics, thermal conductivity and acoustic waves.
\end{abstract}

\section{Introduction}

The main aim of works, results of which are presented in this paper, was to develop a numerical model for enabling investigation of cracks generated acoustic emission (AE) signals occurring in pipelines based on computer simulations. The issue regard to non destructive testing of pipelines for transmitting gaseous chemical substances in temperature below minus $100^{\circ} \mathrm{C}$. Micro-cracks, when present in the material structure, initiates development of discontinuities, which under operational load develop to dominant crack in the fatigue damage and plastic strain process and finally may lead to uncontrolled damage. This in turn causes technical and economic hazard for the production process and environmental protection.

The non-destructive testing method is commonly applied for detection and localization of damages occurring in technical various facilities $[1,2,3]$. Theoretical analyses and computer modeling of defect existing in the structure of pipelines made of steel, on their creation and propagation, were investigated e.g. in [4-8].

The main contribution of this paper lies in presentation of simulation results, performed by using a multiphysical model of a transportation pipe, coupling structural mechanics, thermal conductivity and AE waves. Chosen model parameters were changed and their impact on the achieved results was depicted and discussed.

\section{The numerical model}

\subsection{Modelling assumptions}

The modelling task was performed using the COMSOL Multiphysics environment. It was assumed that the AE wave was emitted at the surface of the pipe due to load created by micro-crack located within its structure. The tube is made of austenitic steel type 304 (A312TP304). For modelling purposes the crack was assumed as heterogeneity in the material structure, which leads to local stress. This stress induces elastic waves which propagate in all directions in the pipe. Reaching the boundary of the pipe, the elastic waves transform to acoustic emission waves, which propagate to the surrounding. It was further assumed that (1) the displacements at the crack tip are small, (2) due to small value of strain, linearization of equations, describing the relationship between the strain and displacement is possible, (3) constant coefficients of the constitutive equations describing the relations between the stress and strain, due to linear elastic type material. The numerical model calculates the distribution of stress and displacement caused by pressure acting on the inner pipe surface, negative gas temperature and the crack presence in the stationary state. The advantage of the applied software lies in the possibility of integration of partial linear differential equations describing the displacement

\footnotetext{
Corresponding author: d.wotzka@po.opole.pl
} 
field in solid bodies, heat flow and acoustic wave equations. The dependent variables are the displacement vector $\mathbf{u}=[u, v]$, temperature $T$ and acoustic pressure $p$. Simulations were performed in the two-dimensional space with axial symmetry. The independent variables in the model include geometric coordinates $x$ and $y$, time $t$ and acoustic wave frequency $f$. The physical parameter values for steel and air are given in Table 1 .

Tab. 1. Physical parameter values of the materials applied.

\begin{tabular}{|l|c|c|}
\hline \multicolumn{1}{|c|}{ Parameter name } & Value & Unit \\
\hline \multicolumn{2}{|c|}{ Steel - within the solid domain } \\
\hline Young's modulus & $211 \mathrm{e} 9$ & $\mathrm{~Pa}$ \\
\hline Poisson ratio & 0.3 & 1 \\
\hline Density & 80300 & $\mathrm{~kg} / \mathrm{m}^{\wedge} 3$ \\
\hline Thermal expansion ratio & $16 \mathrm{e}-6$ & $1 / \mathrm{K}$ \\
\hline Thermal conductivity & 15 & $\mathrm{~W} /\left(\mathrm{m}^{*} \mathrm{~K}\right)$ \\
\hline Heat capacity & 500 & $\mathrm{~J} /(\mathrm{kg} * \mathrm{~K})$ \\
\hline Material type & izotripic & - \\
\hline \multicolumn{2}{|c|}{ Air- within the pressure acoustic domain } \\
\hline Density & 1.225 & $\mathrm{~kg} / \mathrm{m}^{\wedge} 3$ \\
\hline Acoustic wave velocity & 343 & $\mathrm{~m} / \mathrm{s}$ \\
\hline
\end{tabular}

The governing equations used for numerical calculations using FEM are described in detail in an earlier publication [8], in which other analysis results using similar model are depicted.

\subsection{The object under study - geometry}

The object under study is a cross section of pipe of length equal to $50 \mathrm{~mm}$, including axis of symmetry, in center of which the micro crack is located. The resulting model dimension is $6 \times 50 \mathrm{~mm}$. Its geometry is depicted in Fig. 1, where also the applied boundary conditions are presented, which are as follows. For the mechanical domain: free condition at the outer surface of the pipe (edges no 5\&6), displacement condition at the crack tip (point no 4), pressure condition at the inner pipe wall (edge no 2), symmetry condition at the vertical axial symmetry. For the thermal conductivity domain: temperature at edge no 2 , surface to ambient radiation at edge no 5, open boundary at edge no 6 and also symmetry condition at the vertical axial symmetry. The micro crack tip is marked as red dot in the point no 4 .

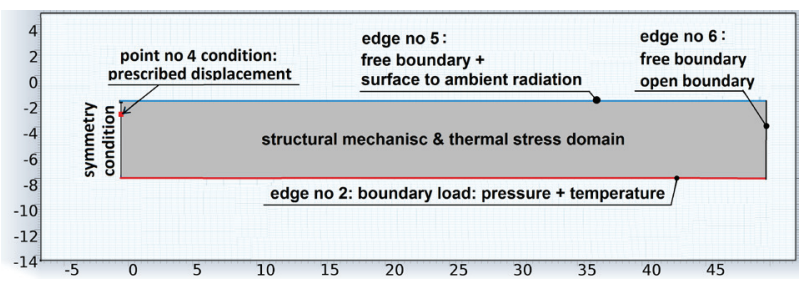

Fig. 1. Presentation of boundary conditions applied in model

Free condition denotes that there is no force and limitations at the boundary of the area. The boundary load means the effect of the pressure of gas pressing the inner pipe wall. Prescribed displacement means a growing crack. The model assumes displacement of 0 towards the pipe interior ( $y$ axis). Such a condition corresponds to the 'fixed'-type condition and stands for displacement $u=0$. Temperature at a boundary means in the considered case the effect of the negative temperature of gas pressing the inner pipe wall. Surfaceto-ambient radiation means the infiltration of ambient heat inside the pipe.

Tab. 2. Range of the analyzed parameter values.

\begin{tabular}{|l|l|l|}
\hline Parameter & Range of the analyzed values & Unit \\
\hline Temperature & $\{88,99,110,121,132\}$ & $\mathrm{K}$ \\
\hline Pressure & $\{2.58,2.91,3.23,3.55,3.88\}$ & $\mathrm{MPa}$ \\
\hline Freuquency & $\{100: 10: 200\}$ & $\mathrm{kHz}$ \\
\hline Time & $\{0: 0.001 / 29: 0.0010\}$ & $\mathrm{s}$ \\
\hline
\end{tabular}

In order to simulate acoustic phenomena the geometry was extended by $10 \mathrm{~mm}$ of surrounding air domain of room temperature and $10 \mathrm{~mm}$ of the so called perfectly matched layer (PML), an area that simulates unrestricted space, as depicted in Fig. 2. The model was applied for determination of the impact of the gas pressure and temperature variations on the dependent values within the analyzed solid domain and for calculation of acoustic pressure and sound power level in the immediate vicinity of the pipe, thus in the air domain. Additionally to the boundaries described earlier, acoustic pressure conditions was set at edge no 5 as source of AE wave, which is a derivative of the 'von Mises' stress at the outer pipe wall. The acoustic wave propagating in PML is not reflected and does not return to its bordering center. A continuity condition is set at the boundary between the PML and acoustic domain, which is fulfilled at all the inner boundaries.

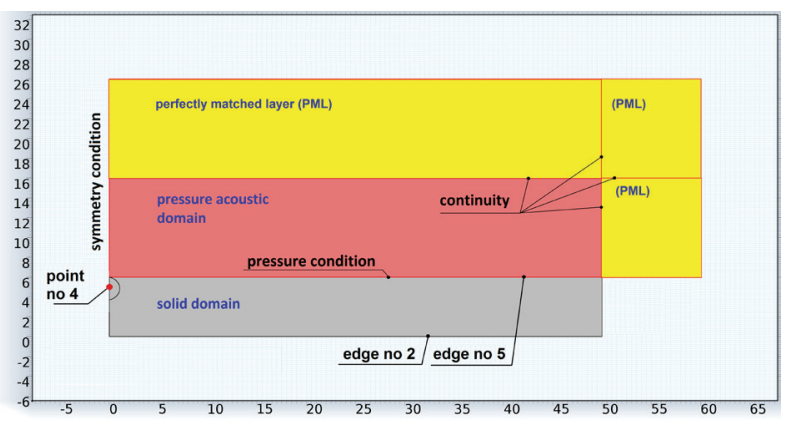

Fig. 2. Boundary conditions regarding the acoustic domain.

\section{Example results}

Fig. 3 present example calculations depicting local distribution of 'von Mises' stress, $\mathrm{N} / \mathrm{m}^{2}$, obtained at the vicinity of the crack tip for $p=3.234 \mathrm{e} 6 \mathrm{MPa}, T=110 \mathrm{~K}$. The greatest decrease in stress values can be observed up to 30 microns from the crack tip on the y axis and up to 10 microns on the $\mathrm{x}$ axis. The maximal calculated stress is at the tip and equals $4.338 \mathrm{e} 10 \mathrm{~N} / \mathrm{m}^{2}$.

Fig. 4 present values of the 'von Mises' stress along edge no 5 (see markings in Fig. 1) for two temperature and five pressure values. In particular, results for two extreme gas temperature values, i.e. $T=88 \mathrm{~K}$ and $T=$ $132 \mathrm{~K}$, and all the considered gas pressure values are presented. 


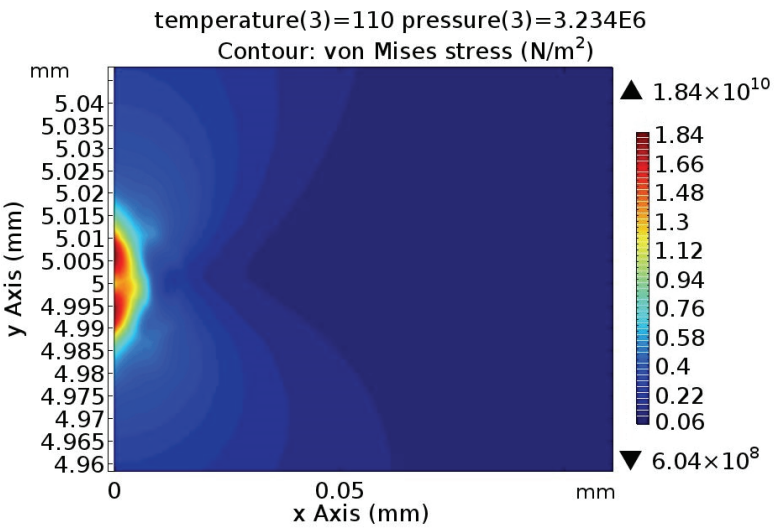

Fig. 3. Values of the 'von Mises' stress in the vicinity of the crack tip.

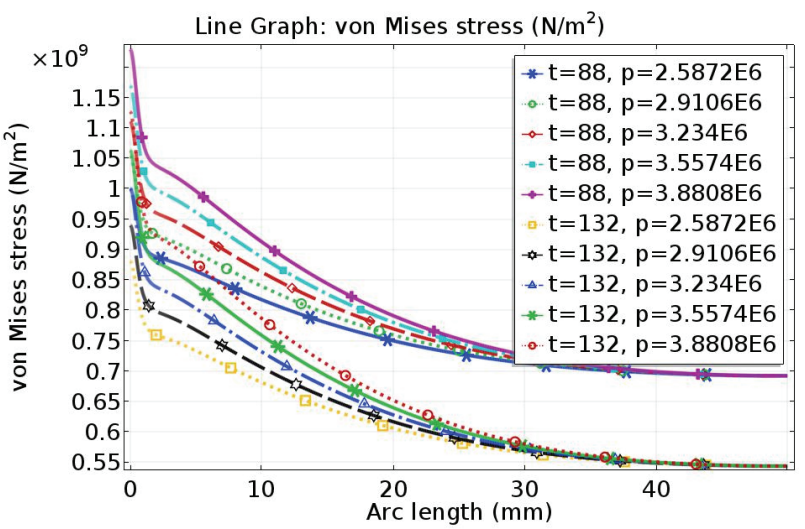

Fig. 4. Values of the 'von Mises' stress along edge no 5 for two temperature and five pressure values.

From the graph it can be recognized that on the outer surface of the pipe (edge no 5) the stress decreases exponentially with increasing distance from the crack tip. The impact of pressure is significant up the distance of about $30 \mathrm{~mm}$. The calculated values do not decrease to zero and at the furthest distance from the crack tip (50 $\mathrm{mm}$ ), the impact of gas pressure disappears. The temperature of the gas transported in the pipe has a significant impact on the calculated stress values, which are higher by lower temperatures.

In Fig. 5 total displacement values obtained along the inner pipe surface (see edge no 2 in Fig. 1) for the same parameters as above mentioned are presented.

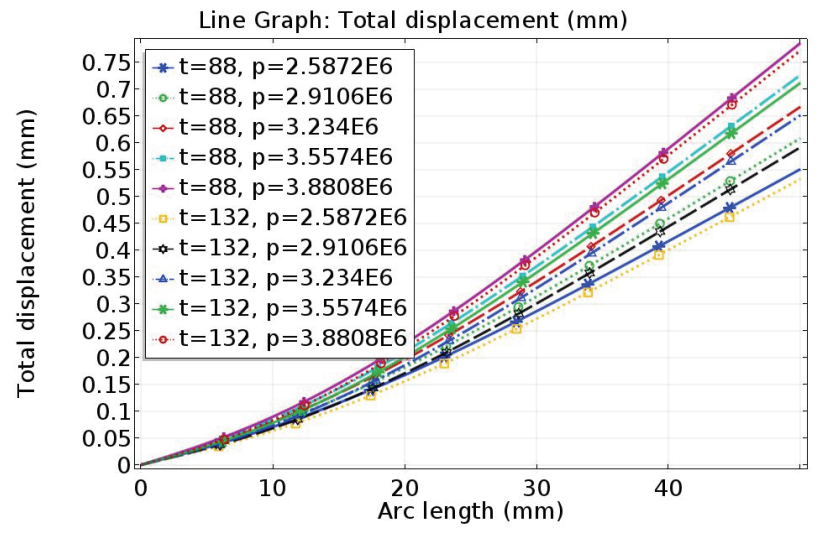

Fig. 5. Values of the displacement along edge no 2 for two temperature and five pressure values.
From Fig 5 it follows that the value of structural displacement increases together with the rising distance from the crack tip. Far from the gap, at a distance of 50 $\mathrm{mm}$, both the impact of the gas pressure and the influence of the temperature are visible, while the latter showing a much lower impact.

In Fig. 6 total acoustic pressure distribution in the considered area is presented. The graph depicts example calculations for sinusoidal $\mathrm{AE}$ wave with a main frequency component equal to $100 \mathrm{~Hz}$. In the PML domain the waveform is completely suppressed.

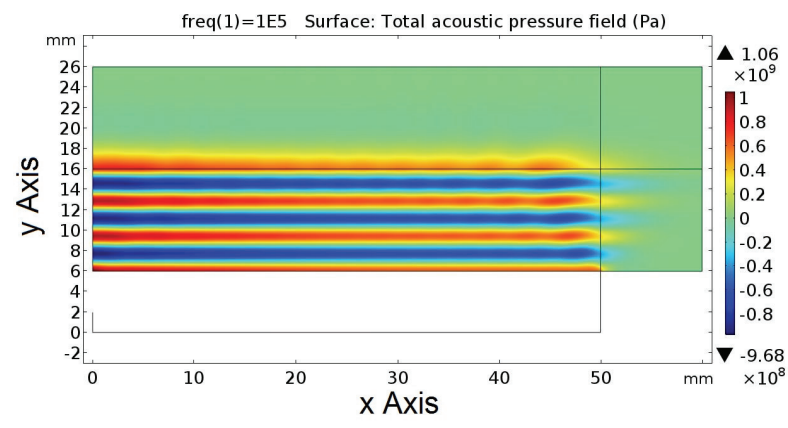

Fig. 6. Distribution of the total acoustic pressure field within the acoustic domain.

In Fig. 7 transient analysis results depicting dynamics of acoustic pressure values, calculated at points $8-12$, which are located across the acoustic domain in its center, are presented. As AE source a Gaussian function, of parameters determined based on experimental measurements, was applied. The moving ridge of the EA wave can be clearly seen.

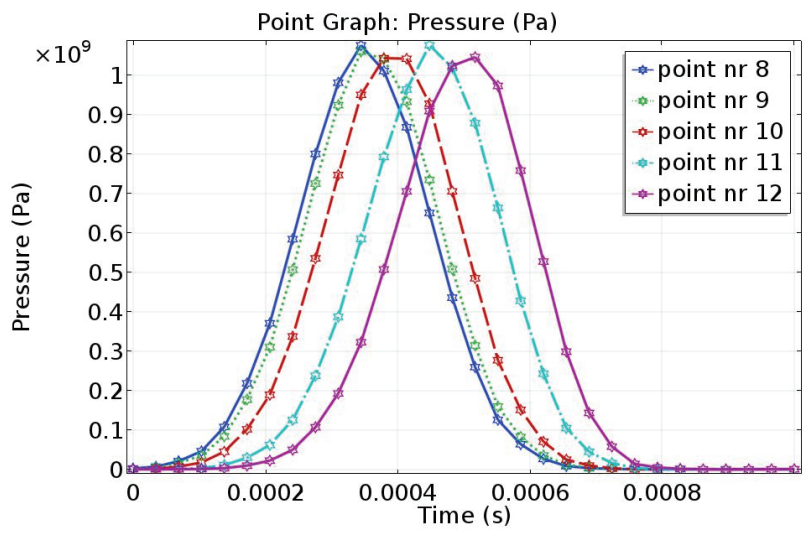

Fig. 7. Values of the acoustic pressure calculated in various points.

\section{Results and discussion}

Space distributions of the 'von Mises' stress, generated in the pipe for gas pressure changes in the range of 3.234 $\mathrm{MPa} \pm 20 \%$ and temperature variations in the range of $110 \mathrm{~K} \pm 20 \%$ and the sound pressure distributions in the air around the pipe were analyzed in the task. Based on the obtained values the following conclusions were driven:

- $\quad$ at the crack tip, the stress is constant and does not depend on the temperature of the gas inside the pipe. On 
the other hand, its value increases proportional to the pressure of the gas transported in the pipe. The changes in the gas pressure have smaller impact as compared to those of the temperature changes;

- $\quad$ on the outer surface of the pipe the stress decreases exponentially with increasing distance from the crack tip. This value does not decrease to zero but stabilizes at a value, which varies from the model parameters. At the points furthest from the crack tip, the difference in gas pressure difference that is visible in the immediate vicinity of the crack tip, disappears. The stress value depends on the temperature of the gas transported in the pipe, this dependence is however not linear relative to distance;

- differences in stress values for the same gas pressure values and higher temperatures are in the crack tip surrounding smaller than at the distance further than $50 \mathrm{~mm}$ from the crack tip;

- along the distance simulating the crack, at a distance of up to $20 \mu \mathrm{m}$ from its tip, the stress value decreases rapidly. On the remaining length, up to the edge of the object, the stress is stabilized. There was also no significant influence of both temperature and gas pressure on the stresses over the entire length of the crack;

- $\quad$ along the edge furthest from edge no 6, the gas pressure does not affect the stress value. On the other hand, its value is determined by the temperature of the gas; the higher the temperature, the less the stress;

- there was no effect of gas pressure on the temperature distribution in the pipe. The temperature range was determined by the temperature of the gas. In addition, there was a slight diffusion of the ambient temperature to the inner structure of the pipe;

- the largest displacements occur at the locations furthest from the crack tip. At a distance of $50 \mathrm{~mm}$ from the tip, the displacement is strongly dependent on the gas pressure and to a much lesser extent on the temperature;

- $\quad$ the sound pressure level in the immediate vicinity of the pipe does not depend on the frequency of the acoustic wave emitted on the outer wall of the pipe and reaches a maximum of $272.46 \mathrm{~dB}$. In contrast, the distribution of total acoustic pressure in the air domain depends on the frequency with which the acoustic wave emits from the source;

- within $10 \mathrm{~mm}$ of the pipe surface there is no noticeable amplitude-damping that exceeds $1 \mathrm{GPa}$. Throughout the length of the analyzed section, the ridges and valleys of the acoustic wave propagating in the domain are clearly visible;

- based on the amount of stress, it is possible to infer where local stresses may cause the development of the cracks and thus the generation of the EA wave. The value of the 'von Mises' parameter may be an indicator of the amplitude of the EA wave.

\section{Conclusions}

This paper is related to improvement of the AE method, applied for detection and localization of defects occurring in transportation pipes. During the theoretical studies COMSOL Multiphysics was used, which made it possible to perform numerical simulations with the Finite Element Method. The aim of the research task was to study the influence of changes in pressure and temperature values of the gas, transported inside a pipeline made of stainless steel, on the values of the stresses, generated in the structure of the pipe, due to a discontinuity, which was modelled in the form of a micro crack. The stress level results from the load acting on the micro crack, and directly affects the intensity of sound pressure, which is emitted during this phenomenon. The task was realized by integrating differential equations describing the field of displacement, conduction of heat in solids and the equations describing acoustic waves, propagating in the air.

The work was founded by The National Centre for Research and Development within the LIDER Project no LIDER/18/44/L-3/NCBR/2012.

\section{References}

1. A. Mostafapour, S. Davoudi, Appl. Acoust. 74 2013)

2. C. Nayak, Int. J. Appl. Eng. Res. 9 (2014)

3. H. Majchrzak, A Cichoń, S Borucki, Arch. Acoust. 42 (2017)

4. J. Abolle Okoyeagu, J. Palacio Torralba, Y. Chen, R. Reuben, Proc. Of 31 Conf. on European Working Group on Acoustic Emission, Fr.3.B.2, Sept. 3-5, Germany, (2014)

5. E. Paffumi, K.-F. Nilsson, Z. Szaraz, Eng. Failure Anal. 47 (2015)

6. C. Ruggieri, F. Dotta, Eng. Struct. 33 (2011).

7. K.R. Jayadevan, E. Berg, C. Thaulow, E. Østby, B. Skallerud, Int. J. Solids Struct. 43 (2006)

8. D. Wotzka, T. Lusa, J. of Mech. Sci. Technol. 29 (2015) 